\title{
Variation of repetitive cortical spreading depression waves is related with relative refractory period: a computational study
}

\author{
Bing Li, Shangbin Chen*, Dong Yu and Pengcheng Li \\ Britton Chance Center for Biomedical Photonics, Wuhan National Laboratory for Optoelectronics, Huazhong University of \\ Science and Technology, Wuhan 430074, China \\ * Correspondence: sbchen@mail.hust.edu.cn
}

Received May 5, 2015; Revised September 11, 2015; Accepted September 28, 2015

\begin{abstract}
Cortical spreading depression (CSD) is an important experimental model for diseases such as stroke, epilepsy and migraine. Previous observations indicated that the amplitude and velocity of the typical direct current potential shift during repetitive CSD waves were varying. The recovery state of the tissue was found related with the variation of successive CSD waves. A computational model in this paper aimed to investigate the role of relative refractory period of CSD. This model simulated that continuous injection of KCl solution induced repetitive CSD waves. The first CSD wave often had a larger amplitude and faster velocity than those of the succeeding secondary waves. The relative refractory period lasted much longer than the recovery of ions turbulence. If the induction interval was long enough for recovery, a series of CSD waves would have the same profile as the first one. In the relative refractory period, an early stimulation might lead to a late initiation of CSD, i.e., "haste makes waste". The amplitude and velocity of CSD waves were found increasing with the initiation interval and asymptotic to those of the first CSD wave. This study verified that the propagation dynamics of CSD waves is modulated by the relative refractory period. It suggested that the refractory period is critical for preventing undesirable CSD waves.
\end{abstract}

Keywords: cortical spreading depression; time-varying; relative refractory period; computational study

\section{INTRODUCTION}

Cortical spreading depression (CSD) was first observed in the cerebral cortex of rabbits by Leão in 1944 [1,2]. CSD is a pathophysiological phenomenon characterized by a transient suppression of neuronal activity, failure of ion homeostasis, a negative direct current (DC) potential shift and the depression of electrocorticogram [3-6]. During CSD, oxygen consumption and local cerebral blood flow will increase [7], and the cerebral vessel will change [8]. CSD is suspected to be involved in migraine [9-11], and have an underlying role in epilepsy $[6,12,13]$ and stroke $[14,15]$. Different kinds of methods, including mechanical injury, chemical or anoxic stimuli [16-20], have been used to initiate CSD in animal brain to study the mechanisms of CSD. However, the mechanisms of CSD are still not completely clear $[6,21,22]$.

CSD has been observed with electrophyiological, ionic and optical changes spreading in cortex [23]. Characterizing propagation dynamics of CSD may help to understand its unclear mechanisms. Here, we are focusing on the typical ionic changes of potassium and calcium. Previously, two types of propagation events had been recorded during the repetitive $\mathrm{CSD}$ induced by $\mathrm{KCl}$ $[18,24]$. The primary event was the first wave propagating through the cortex, while the secondary events were the succeeding waves. These two kinds of events had similar duration, but some of the propagation patterns such as amplitude and velocity were different $[18,24,25]$. This implied that the primary event can modulate the following events, but the nature of the modulation is still not known [26]. Our work has demonstrated that the elicitation interval of CSD waves has effect on the spatiotemporal evolution of CSD $[27,28]$. Independent on the stimulation of pinprick or $\mathrm{KCl}$, a short interval of the current CSD to the last CSD no more than 4 min would induce the current 
CSD be partially propagated [28]. Thus, CSD could not be an "all or none" phenomenon [25]. This challenged the traditional conception.

Usually, the different regional manifestation of CSD was thought as the different cytoarchitecture and glia/ neuron ratio $[5,6,29,30]$. However, the temporal variation of propagation patterns of successive CSD waves in the same region could not be entirely accounted by the cytoarchitecture. In fact, the modulation of the refractory period of CSD was found involved in the variation [3133]. Both the experimental and theoretical work addressed the mechanisms of gap junctions, glial cells (astrocytes) and ion channels. The simulation results revealed the refractoriness conditions of CSD (i.e., the slowly inactivating inward currents overcome the outward ones) [32,34]. In order to bridge the gap between the macroscopic and microscopic level of CSD, this study paid more attention to study the variation of CSD patterns under long-lasting and transient stimulation conditions. This would be helpful to control the stimulation condition so that the uniform CSD wave is induced for practical requirement [35]. Correspondingly, a phenomenological model was introduced in this paper.

\section{RESULTS}

\section{Dynamics of ionic concentrations during a single CSD wave}

After a transient injection of $\mathrm{KCl}$ with concentration of $20 \mathrm{mM}$, only one CSD was evoked in the modeling system. The changes of ionic concentrations accompanied CSD were shown in Figure 1: $\left[\mathrm{K}^{+}\right]_{\mathrm{o}}$ rose up to $60 \mathrm{mM}$, $\left[\mathrm{Ca}^{2+}\right]_{\mathrm{a}}$ up to $0.17 \mathrm{mM},\left[\mathrm{Ca}^{2+}\right]_{\mathrm{n}}$ up to $0.13 \mathrm{mM}$, and $\left[\mathrm{Ca}^{2+}\right]_{\mathrm{o}}$ down to $0.33 \mathrm{mM}$. All these 4 simulated ionic changes were consistent with the experimental records $[5,36]$. The duration of $\left[\mathrm{K}^{+}\right]_{\mathrm{o}}$ change was about $100 \mathrm{~s}$ (measured at half of the maximum amplitude), then it returned to the resting state within $3 \mathrm{~min}$. We focused on three sites $(3 \mathrm{~mm}, 7 \mathrm{~mm}, 11 \mathrm{~mm}$ from the injection center respectively), acting as probes in the simulation to calculate the velocity of CSD. The CSD propagated
A

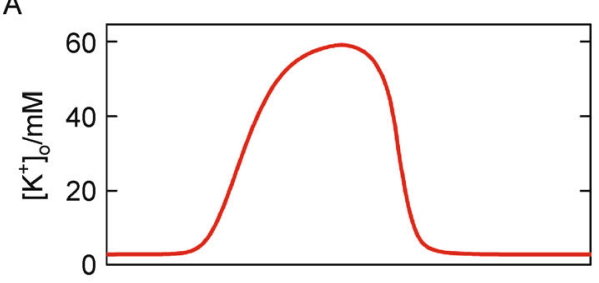

C

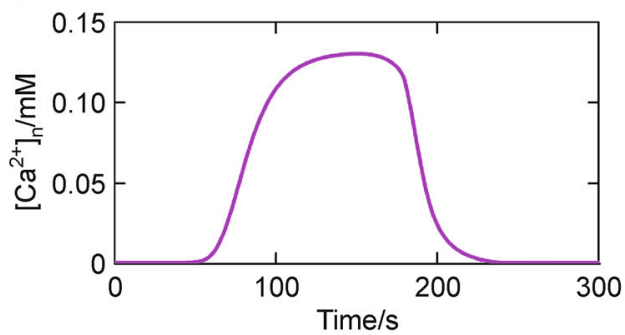

B

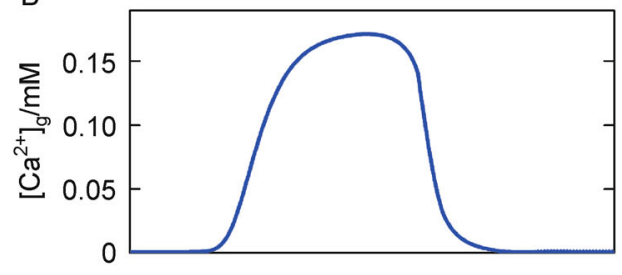

D

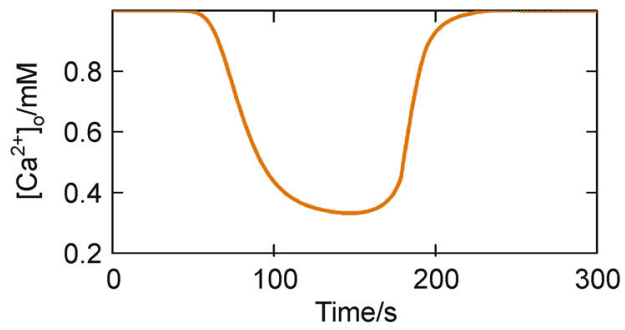

E

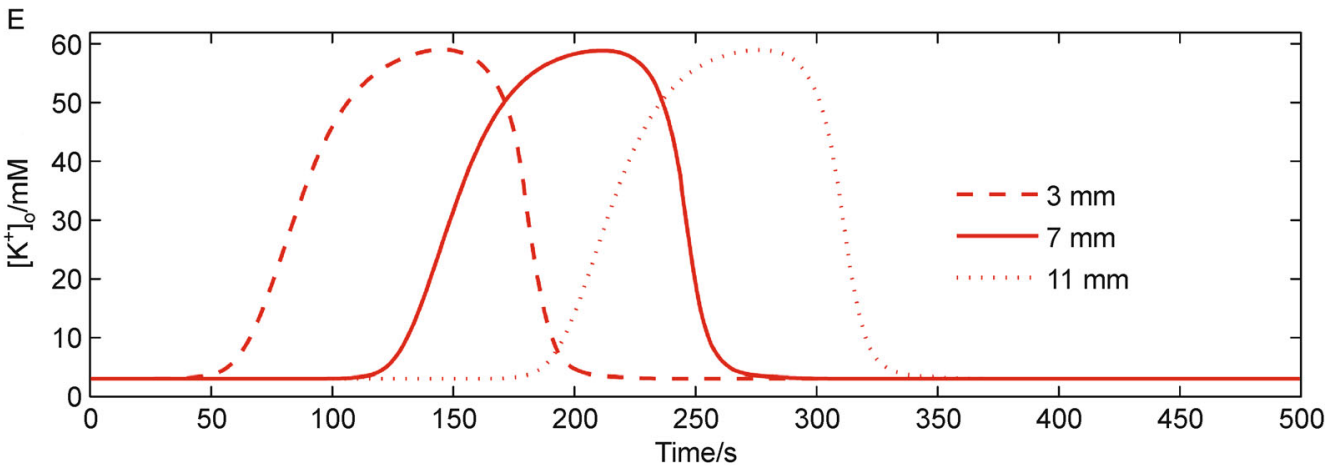

Figure 1. Dynamics of ionic concentrations after $1 \mathrm{~s}$ transient, $20 \mathrm{mM} \mathrm{KCl}$ injection. (A) is the potassium concentration in the extracellular space, and (B-D) is calcium concentration in the astroctyes, neurons and extracellular space, respectively. In (E), the time courses of $\left[\mathrm{K}^{+}\right]_{\mathrm{o}}$ are from 3 different sites with $3 \mathrm{~mm}, 7 \mathrm{~mm}$ and $11 \mathrm{~mm}$ away from the injection center. 
through the cortex at a speed of $3.7 \mathrm{~mm} / \mathrm{min}$ in this model, which was similar to the experimental data $[5,6]$. In Figure 2, a continuous $\mathrm{KCl}$ injection lasting an hour could bring a series of time-varying CSD waves in the region of interest. This phenomenon mimicked the experiments $[18,25,27]$. Both the transient and sustained stimulation results validated the current model.

\section{Temporal evolution of repetitive CSD waves}

The modeling results of repetitive CSD waves also reproduced that the amplitude of the primary event was larger than the average of the secondary events [26]. The first wave of $\left[\mathrm{K}^{+}\right]_{\mathrm{o}}$ could rise nearly to $60 \mathrm{mM}$, while the average peak value of the following waves in an hour was just about $38 \mathrm{mM}$, decreasing by $22 \mathrm{mM}$. The profile of the primary wave during the repetitive CSD waves was similar to that of the single one in our modeling [27,37]. The amplitudes of the secondary events did not decrease monotonously but varied with time (Figure 2). The amplitudes of the several subsequent waves were always smaller than that of the primary one. In addition, the primary CSD wave had a speed of $3.7 \mathrm{~mm} / \mathrm{min}$ and the average speed of the secondary CSD waves was just 1.7 $\mathrm{mm} / \mathrm{min}$. But the duration of the primary wave was similar to that of each succeeding CSD wave [24]. With careful inspection and calculation, a longer time interval after the last CSD would give birth to the following CSD with larger amplitude and faster velocity in this model $[31,32]$. In fact, the primary wave was found propagating through the entire cortex but the following waves might bypass some area of the cortex in experiments [18,26-
28]. This suggested the temporal evolution of CSD was related with spatial evolution: a CSD wave owning large amplitude could propagate more wide area of cortex. The spatiotemporal evolution of CSD waves was related with the time interval between CSD waves [27]. This modeling work verified this point.

\section{The dominating factor of varying CSD waves}

Here, we attempted to seek which factor is dominated to the varying CSD waves in the modeling work. All the six variables during the first two CSD waves were plotted in Figure 3. Even all of the ions involved in this model returned to the resting state, but $R$ was still in the excitement. It hinted that $R$ might have a long-lasting influence on the propagation dynamics of CSD waves. The simulation experiment was performed to validate our conjecture in Figure 4. In the case that $R$ did not recover to the resting state, the following CSD would not have the same profile as the previous one (Figure 4A). More further away for $R$ returning to the resting state made a larger difference between the following CSD and the previous one. When $R$ went back to the normal nearly, the following CSD would have the same profile as the previous one (Figure 4B). Since $R$ represents as the recovery mechanism, $R$ could be a quantitative variable for the relative refractory period.

\section{Relative refractory period and variation of CSD waves}

Two events of transient injection of $\mathrm{KCl}$ with different

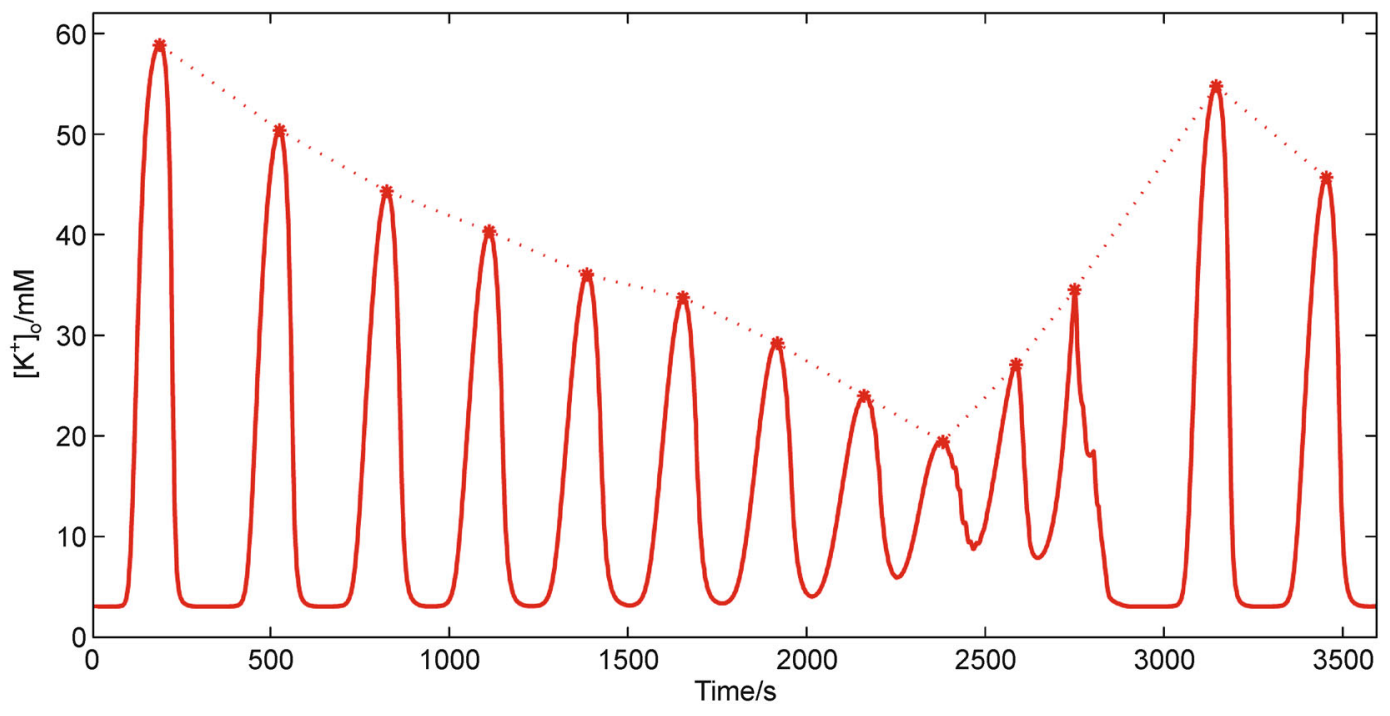

Figure 2. Typical simulated temporal evolution of repetitive CSD waves from $1 \mathrm{~h}$ continuous, $20 \mathrm{mM} \mathrm{KCl} \mathrm{injection} \mathrm{in} \mathrm{the}$ model. The peak amplitudes of $\left[\mathrm{K}^{+}\right]_{\mathrm{o}}$ of $13 \mathrm{CSD}$ waves varies obviously in an hour. The dashed line outlines the changing peak amplitudes of $\left[\mathrm{K}^{+}\right]_{\mathrm{o}}$. 

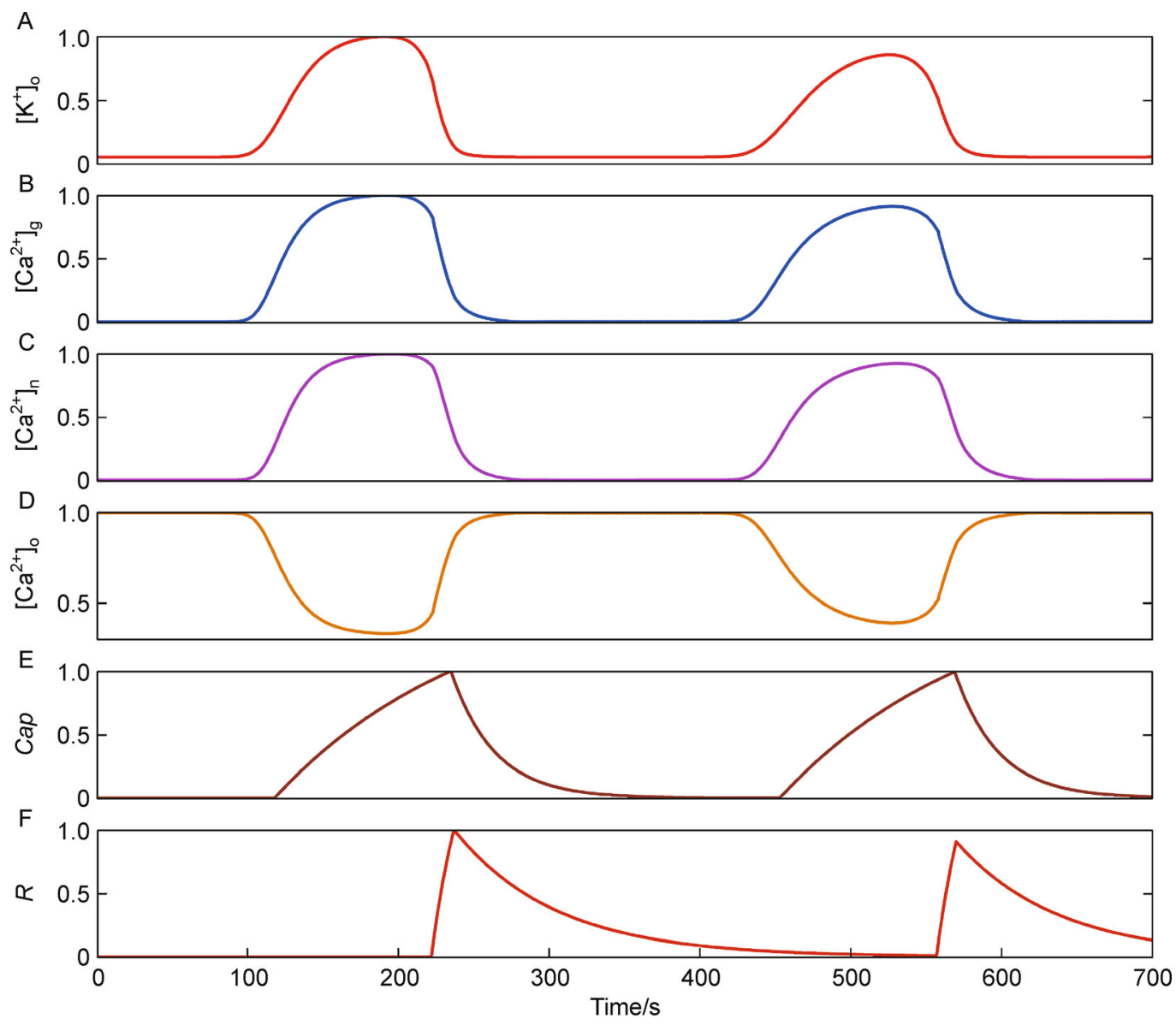

Figure 3. Simulated dynamics of 6 variables during 2 successive CSD waves. The elicitation conditions are the same of Figure 2. Normalized dynamic changes of 6 variables are shown during the first two CSD waves. After the fully recovery of all the 4 ionic changes from the first CSD wave, only $R$ is still in the excitement before the second CSD occurs.

time intervals during the recovery period were modeled to observe the influence of refractory period on the propagation dynamics of $\mathrm{CSD}$ waves. The first $\mathrm{KCl}$ injection happened soon after the simulation. The second stimulation intervals were $240 \mathrm{~s}, 300 \mathrm{~s}, 360 \mathrm{~s}, 420 \mathrm{~s}, 480 \mathrm{~s}$ and $600 \mathrm{~s}$, respectively. It appeared that if the stimulation interval was too short [5], CSD could not be evoked (Figure 5A). The reason was that the stimulation was made during the absolute refractory period. This also indicated that the change of variable $R$ covered the absolute and relative refractory period. An unexpected phenomenon was observed that a later injection of $\mathrm{KCl}$ could induce an earlier appearance of CSD (Figure 5BE). The CSD occurring later had a little faster velocity and a little larger amplitude (Data are list in Table 1). When the stimulation interval was long enough, for example, $600 \mathrm{~s}$ in this model, the secondary events had nearly the same amplitude and velocity as the primary event (Figure $5 \mathrm{~F})$. Ten minutes should be considered as the time that the tissue needs to recover from CSD. It seemed that long interval could reduce the difference among the repetitive CSD waves.

In the above work, there seemed to be a positive correlation between the amplitude and the velocity during the repetitive CSD waves. Here, a CSD was evoked by transient stimulation at the beginning of the simulation. Another succeeding stimulation injection was carried out at different time points from about $300 \mathrm{~s}$ to $1000 \mathrm{~s}$ to the first stimulation. Within $290 \mathrm{~s}$, we did not give any stimulation because no CSD can be induced during the absolute refractory period. For each trial, there were two CSD waves induced. The amplitude and velocity of the second CSD wave were recorded at a location $7 \mathrm{~mm}$ from the injection center. The time interval of the peak values of the two CSD waves called peak interval was also recorded there. In Figure 6, it appeared that the amplitude of CSD had the same trend as the velocity [32]. The larger the amplitude was, the faster the velocity. A decline at the beginning of the curves was noticed. It suggested that under a certain time frame during the relative refractory 


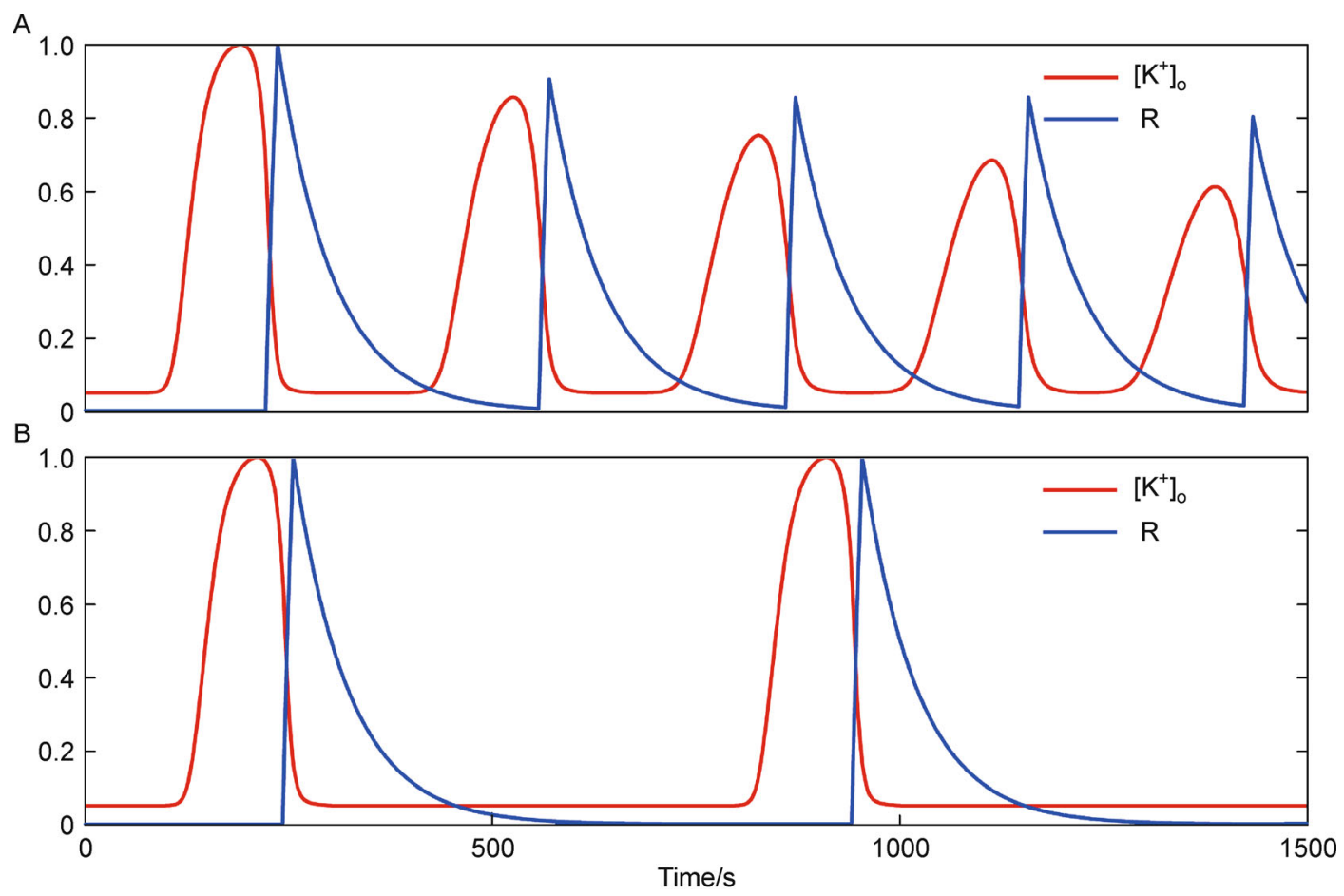

Figure 4. Effect of $R$ on the propagation dynamics of CSD waves in the model. In (A), the results are truncated from the first 1500 s duration of the simulation in Figure 2. Due to the non-resting $R$, there is large difference among the CSD waves. In (B), the results are produced by two transient stimulations $(1 \mathrm{~s}, 20 \mathrm{mM} \mathrm{KCl}$ injection) with an interval of $700 \mathrm{~s}$. Without the influence of $R$, the two successive CSD waves have the same profile. All of the parameters are normalized in $(A-B)$.

period, an early injection could evoke a CSD having a little faster velocity as well as a little larger amplitude. From $350 \mathrm{~s}$ to $600 \mathrm{~s}$, there was nearly a linear relationship between the amplitude and velocity of CSD. About $600 \mathrm{~s}$ later, the amplitude and the velocity changed little, and reached a stable state. CSD evoked at that stable state had almost the same profile as the first CSD wave. Here it suggested that it cost about 10 minutes for the tissue to recover from CSD completely.

In Figure 7, the second CSD could not be observed at the location $7 \mathrm{~mm}$ from the injection center until $400 \mathrm{~s}$ after the first CSD was observed. As peak interval increased, the amplitude of CSD got larger and the velocity became faster. Also, the amplitude and velocity would reach a stable state after $600 \mathrm{~s}$. It was found that it was not a linear relationship between stimulation interval and peak interval in Figure 8. During the relative refractory period, a short stimulation interval might lead to a long peak interval. The longer peak interval meant that the second CSD occurred much later. So, a testable prediction came into being that under a certain time window during the relative refractory period, an earlier injection could evoke a later appearance of CSD, and the CSD occurring later had a little faster velocity as well as a little larger amplitude. This demonstrated that haste makes waste.

\section{DISCUSSION}

Since the clinical relevance of CSD, there has been extensive interest in both experimental and computational aspects of CSD [6,13,32,38-42]. This paper aimed to simulate the variation of repetitive CSD waves found in experiments with a computational study $[18,25,27,28]$. It was different from some previous theoretical work that addressed the characteristics of a solitary CSD wave [4345]. Many experiments showed the inhomogeneous spatiotemporal evolution of successive CSD waves, but the modeling work on this issue was still few [32,33]. The present modeling has been successful in simulating the single or successive CSD waves. Also, this work has shown that the variation of repetitive CSD waves is related with the relative refractory period. Since the current model was only one spatial dimension, the variation of CSD was mainly shown as temporal ionic changes. The spatiotemporal dynamics had been qualitatively studied in our previous work [33].

In this study, $R$ represented as the recovery mechanism, especially standing for relative refractory period. It was shown that $R$ lasts a longer duration than the extracellular ion disturbance. This suggested that even all of ions go back to the resting state, the tissue is not completely recovered from the last CSD wave with non-resting $R$ 
A

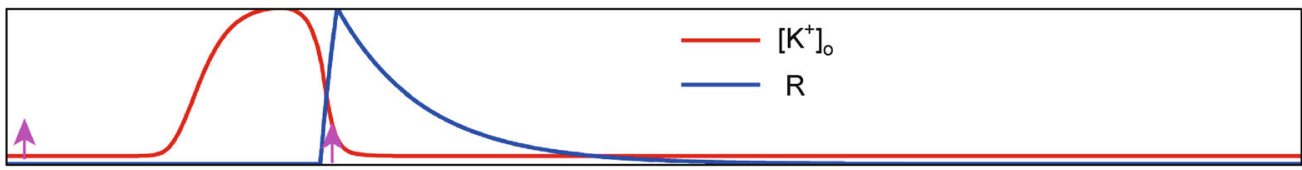

B
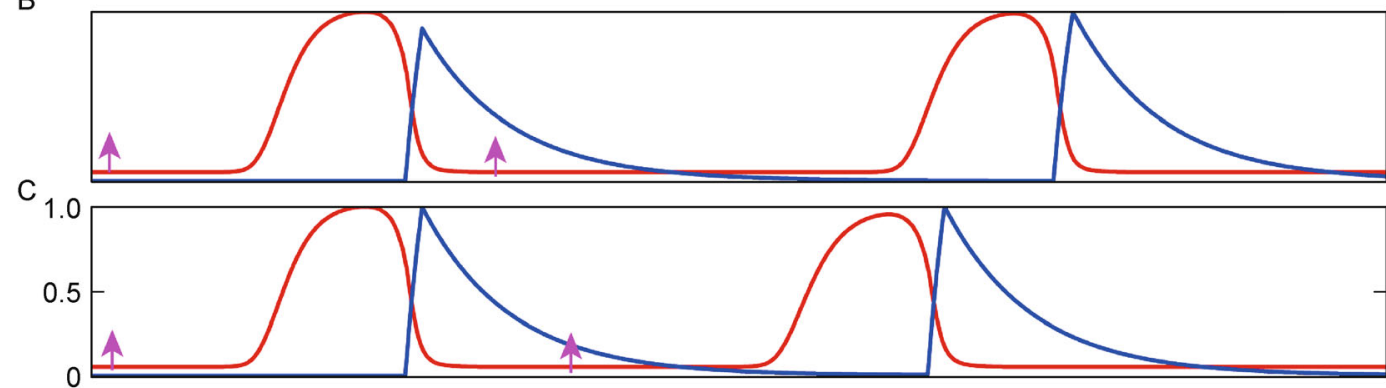

D

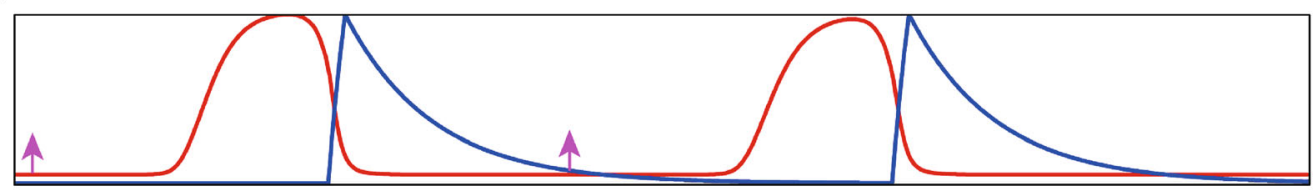

E

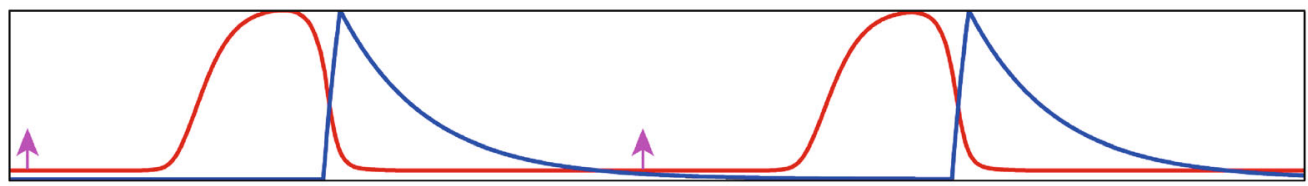

$\mathrm{F}$

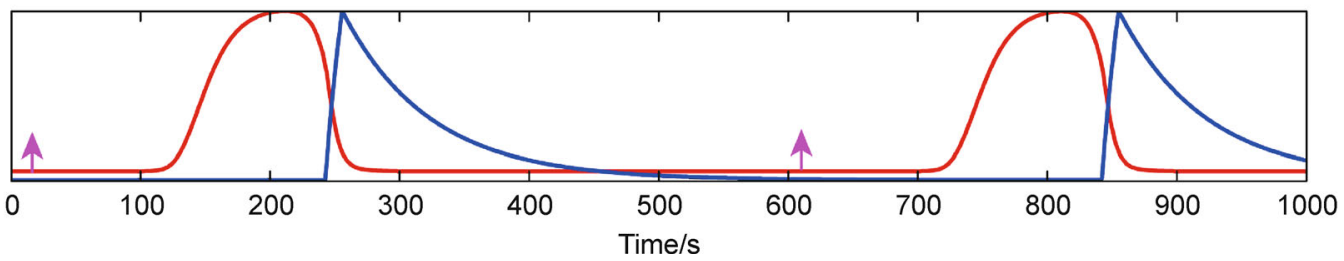

Figure 5. Effect of time interval of stimulation on the propagation dynamics of CSD waves in the simulation. In the six simulated trials $(A-F)$, each stimulation is $1 \mathrm{~s}$ transient, $20 \mathrm{mM} \mathrm{KCl}$ injection but with different time interval to the first stimulation. In $(A)$, no CSD occurs after the second injection. In (B-E), a later injection (including (C-E)) can induce an earlier appearance of CSD than that of $(B)$. This indicates that haste makes waste. In (B-F), the CSD occurring later has a little faster velocity and a little larger amplitude. Each up arrow represents as one injection of $\mathrm{KCl}$. All of the parameters are normalized in $(\mathrm{A}-\mathrm{F})$.

Table 1. Effect of the time interval on CSD.

\begin{tabular}{llll}
\hline $\begin{array}{l}\text { Time interval } \\
\text { of the two } \\
\text { injections/s }\end{array}$ & $\begin{array}{l}\text { *Time interval of } \\
\text { the peaks of } \\
\text { CSD/s }\end{array}$ & $\begin{array}{l}\text { Velocity } \\
/\left(\mathbf{m m} \cdot \mathbf{m i n}^{-\mathbf{1}}\right)\end{array}$ & $\begin{array}{l}\text { Amplitude } \\
/ \mathbf{m M}\end{array}$ \\
\hline 240 & Null & Null & Null \\
300 & 500 & 3.56 & 58.22 \\
360 & 403 & 3.29 & 56.13 \\
420 & 435 & 3.43 & 57.17 \\
480 & 485 & 3.56 & 58.05 \\
600 & 598 & 3.64 & 58.68 \\
\hline
\end{tabular}

*Recorded at a location $7 \mathrm{~mm}$ from the injection center.

value. This was in accordance with the previous study about the refractoriness $[31,32]$. So, it was easy to control the variable of $R$ to modulate the successive CSD waves.
By adjusting some parameters, the variable of $R$ were also found related with the amplitude and frequency of CSD waves (Results not shown). This was consistent with the experimental observation with pharmacological blockers $[31,46]$.

An interesting result of this model was that an earlier stimulation during relative refractory period could induce a later appearance of CSD, i.e., "haste makes waste". This should be a testable prediction in the future experiments. The amplitude and velocity of CSD wave were influenced by the stimulation interval. Further, they had a positive correlation with the peak time interval (i.e., occurrence interval). The amplitude had the same trend as the velocity. This point was similar with previous studies $[31,32,44]$. But the previous work did not distinguish the stimulation interval and occurrence interval (i.e., peak 


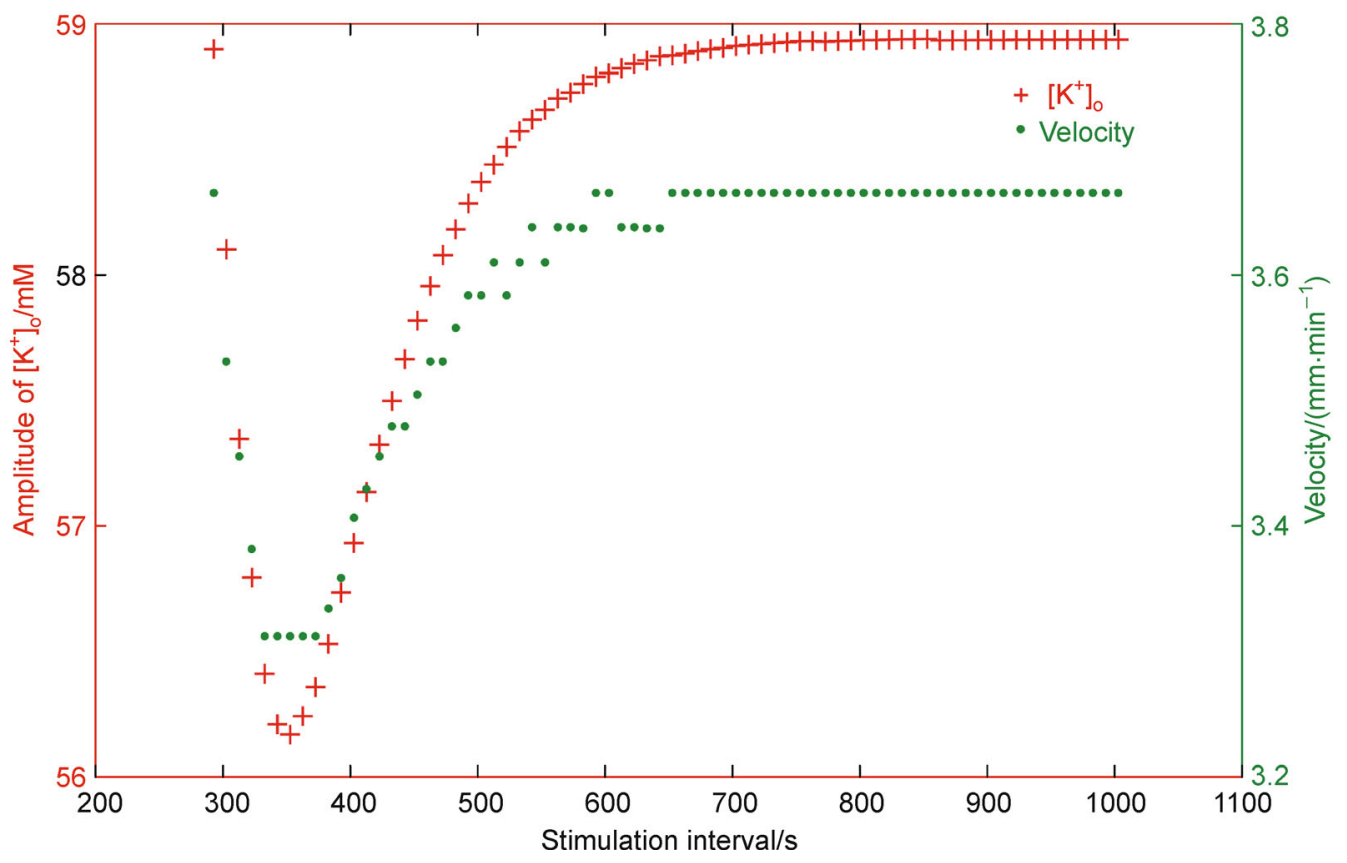

Figure 6. Amplitude and velocity of CSD waves under different stimulation interval. Here, each stimulation is $1 \mathrm{~s}$ transient, $20 \mathrm{mM} \mathrm{KCl}$ injection. There is an obvious positive correlation between amplitude and velocity. When the time interval is longer than 10 minutes, amplitude and velocity of CSD waves reach a stable state. The ' + ' in red represents as amplitude, and '.' in green represents as velocity.

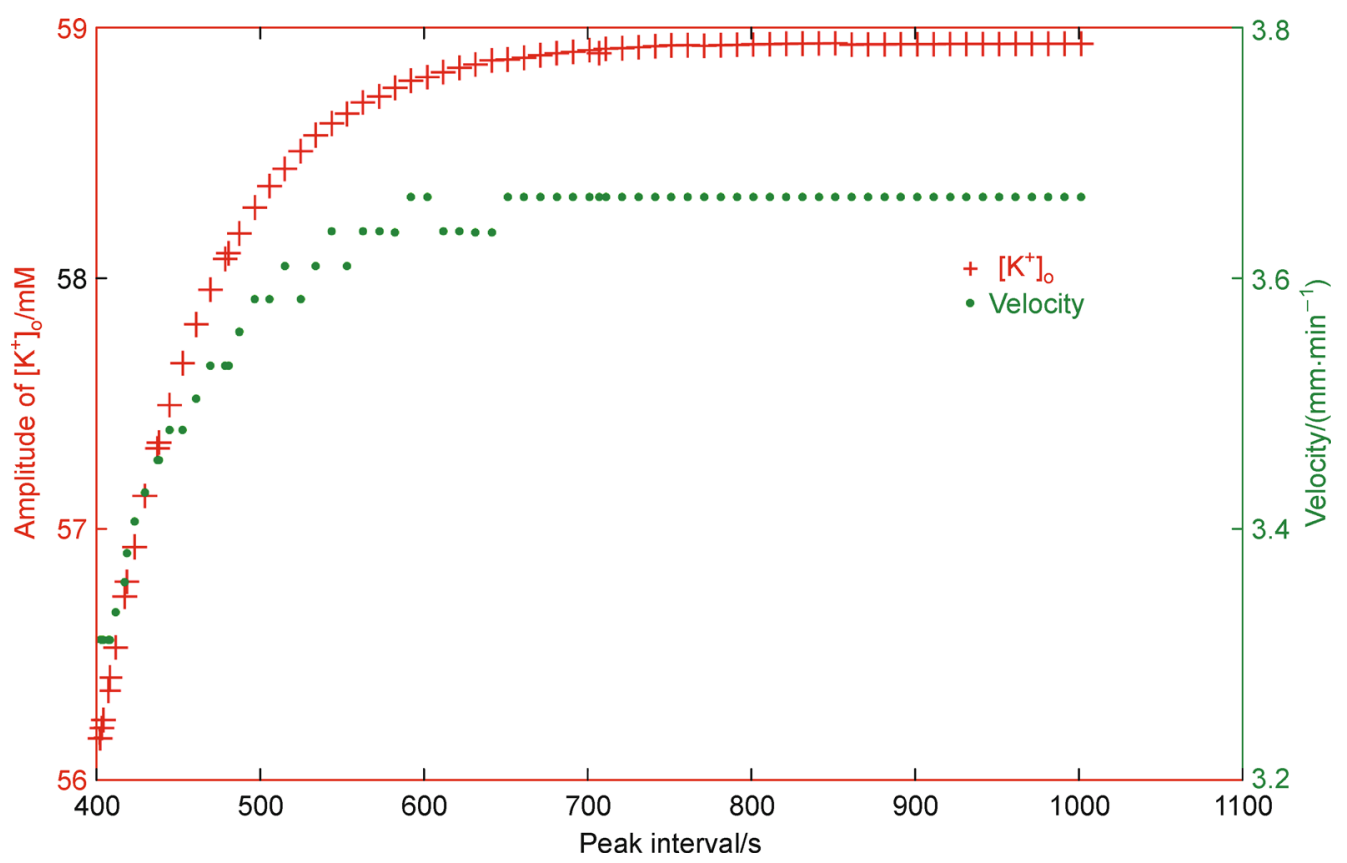

Figure 7. Amplitude and velocity of CSD waves corresponding to the time interval observing the peaks of the two CSD waves. The conditions for CSD induction are the same with those in Figure 6 . There is an obvious positive relationship between amplitude and velocity. The ' + ' in red represents as amplitude, and ' '. in green represents as velocity. 


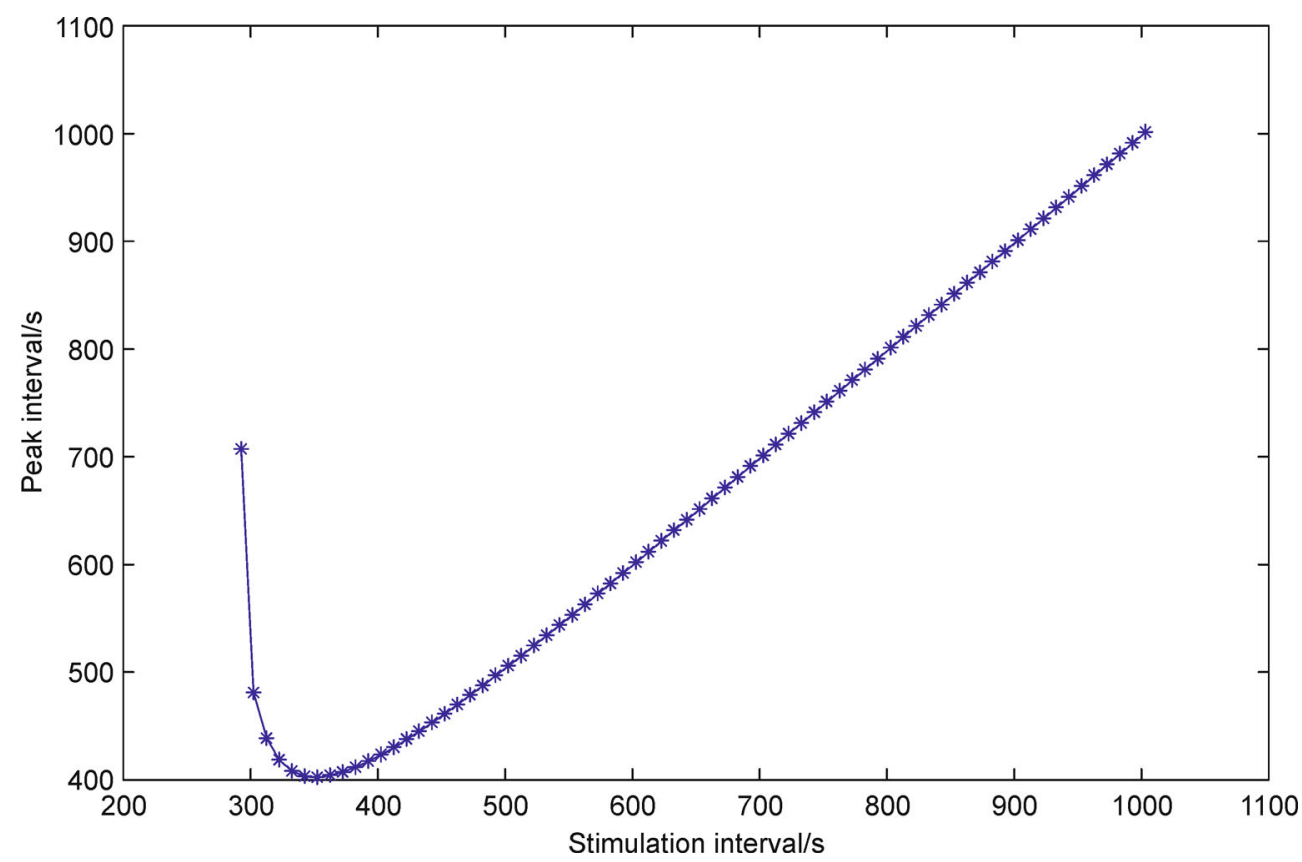

Figure 8. Relationship between the stimulation interval and peak interval of two successive CSD waves. Here, each stimulation is $1 \mathrm{~s}$ transient, $20 \mathrm{mM} \mathrm{KCl}$ injection. The peak interval is not linearly depended on the stimulation interval. At the beginning of the relative refractory period, an early injection of $\mathrm{KCl}$ induced a later occurrence of CSD wave.

interval in Figures 7 and 8). The computational results showed that the amplitude and velocity of successive CSD waves were varying. It inferred that CSD is not necessarily an "all or none" phenomenon. Noticed from the experimental results, the CSD wave had larger amplitude and higher velocity would spread to more wide area of cortex $[18,25,27,28]$. If the uniform CSD wave was required under practical consideration [35], a long enough recovery time about 10 minutes were suggested here.

This model could be used to study some other factors that could influence the propagation dynamics of the repetitive CSD waves, such as gap junction and astrocytes $[47,48]$. However, the current work was mainly a phenomenological model without accurate knowledge of cytoarchitecture and ion channels of cortex. This was not a cellular level model of CSD. The current model had limitations to reflect the individual neurons and glial cells. Neither the function of sodium channels nor the changes of sodium ions were not included yet. In fact, we paid more attention to the rational phenomenal results than the completeness of the model formation. This work suggested that the refractory period is critical for preventing undesirable CSD waves. To the best of our knowledge, the philosophy of "haste makes waste" is first predicted from the proposed model. Taken together, this macroscopic level study of CSD had been proved helpful to modulate and understand the spatiotemporal evolution of CSD waves.

\section{METHODS}

\section{The conceptual model}

We incorporated part of the model from Chapuisat [49] and Tuckwell [38], forming a one dimensional continuum model presented here (Figure 9A). Three compartments in the cortical tissue (gray matter) were considered. They were neurons, glial cells and the extracellular space. Here, blood vessels were not included in this model. Since astrocytes play an important role in CSD $[39,47]$, we took glial cells as astrocytes to simplify the model. The model consists of two ionic species, the potassium and the calcium. $\left[\mathrm{K}^{+}\right]_{\mathrm{o}},\left[\mathrm{Ca}^{2+}\right]_{\mathrm{a}},\left[\mathrm{Ca}^{2+}\right]_{\mathrm{n}}$ and $\left[\mathrm{Ca}^{2+}\right]_{\mathrm{o}}$ respectively represent as the extracellular potassium concentration, the astrocytic calcium concentration, the neuronal calcium concentration, and the extracellular calcium concentration. We did not take the change of the volume of the three compartments into account during CSD. The volume of each compartment was kept as constant. We considered the fraction of extracellular space as 0.2 [50], and the fractions of neurons and astrocytes are $0.07,0.73$ respectively [33]. Our model complies with such following physiological processes (Figure 9B). The stimulation is injection of $\mathrm{KCl}$ solution to induce an initial increase of $\left[\mathrm{K}^{+}\right]_{\mathrm{o}}$. After injecting $\mathrm{KCl}$, the potassium concentration in the extracellular space $\left[\mathrm{K}^{+}\right]_{\mathrm{o}}$ rises. The increase of $\left[\mathrm{K}^{+}\right]_{\mathrm{o}}$ causes all the surrounding cells to depolarize. This makes the voltage-gated calcium channels in astrocytes open, 
leading calcium enter astrocytes and $\left[\mathrm{Ca}^{2+}\right]_{\mathrm{a}}$ increase. Calcium in the astrocytes propagates through gap junctions between astrocytes. Some of calcium enters neurons, making $\left[\mathrm{Ca}^{2+}\right]_{n}$ increase and open calciumdependent potassium channels. Thus, the potassium in neurons goes out to the extracellular space, which is helpful to the increase of $\left[\mathrm{K}^{+}\right]_{\mathrm{o}}$. When the $\left[\mathrm{K}^{+}\right]_{\mathrm{o}}$ is higher than certain threshold, CSD may be induced and propagate. During CSD, the ion homeostasis is interrupted and undergoing a large fluctuation. A few minutes later, the cortical tissue returns to the resting state without successive stimulation.

\section{The equations and the parameters}

The model is made up of two partial differential equations, three ordinary differential equations and an algebraic equation. The Equations 1-4 control the ionic movements, and the variables $C a_{a}, C a_{n}, C a_{o}$ and $K_{o}$ represent the differences of corresponding ionic concentrations at certain time to the resting state. So the initial values of them are zero. Cap in Equation 5 denotes the capacity of cells to recover from the CSD and $R$ in Equation 6 stands for the recovery mechanism, especially for relative refractory period.

$$
\begin{gathered}
\frac{\partial C a_{a}}{\partial t}=D_{C a_{a}} \nabla^{2} C a_{a}+v_{a} K_{o}-g_{a}\left(C a_{a}-C a_{n}\right) \\
-f_{a} \tanh \frac{C a_{a}}{C a_{a}{ }^{0}}-k_{a} C a_{a}{ }^{2}-r_{a} R C a_{a} . \\
\frac{\partial C a_{n}}{\partial t}=g_{n}\left(C a_{a}-C a_{n}\right)-f_{n} \tanh \frac{C a_{n}}{C a_{n}{ }^{0}}-k_{n} C a_{n}{ }^{2}-r_{n} R C a_{n} .
\end{gathered}
$$

$$
\begin{gathered}
C a_{o}=-\frac{\gamma}{\alpha} C a_{a}-\frac{\beta}{\alpha} C a_{n} . \\
\frac{\partial K_{o}}{\partial t}=D_{K_{o}} \nabla^{2} K_{o}+v_{n} C a_{n}-f_{o} \tanh \frac{K_{o}}{K_{o}{ }^{0}}-k_{o} K_{o}{ }^{2}-r_{o} R K_{o} . \\
\left\{\begin{array}{l}
\frac{\partial C a p}{\partial t}=\frac{1-C a p}{T_{0}}, \text { if } C a_{a}>c a \text { or } C a_{n}>c n \text { or } K_{o}>c k \\
\frac{\partial C a p}{\partial t}=-\frac{C a p}{T_{f}}, \text { if } C a_{a} \leqslant c a \text { and } C a_{n} \leqslant c n \text { and } K_{o} \leqslant c k
\end{array}\right.
\end{gathered}
$$

Here $c a, c n$ and $c k$ are thresholds related to the recovery from CSD. Biological meanings and values of the parameters are listed in Table 2.

$$
\left\{\begin{array}{l}
\frac{\partial R}{\partial t}=\frac{1-R}{t_{0}}, \text { if } \text { Cap }>c c a p \\
\frac{\partial R}{\partial t}=-\frac{R}{t_{f}}, \text { if } C a p \leqslant c c a p
\end{array}\right.
$$

Here ccap is the threshold related to the opening of the recovery mechanism. We fixed part of values of the parameters in this model following the algorithm put forward by Chapuisat $[40,49]$.

\section{Implementation}

All the computations and visualizations of the model were implemented in the Matlab environment (Matlab7.0, the MathWorks Inc., USA). An explicit difference method was used to solve the differential equations $[33,51]$. The computations were simulated in a one-dimensional region of the cortex [52]. The time and the space were divided
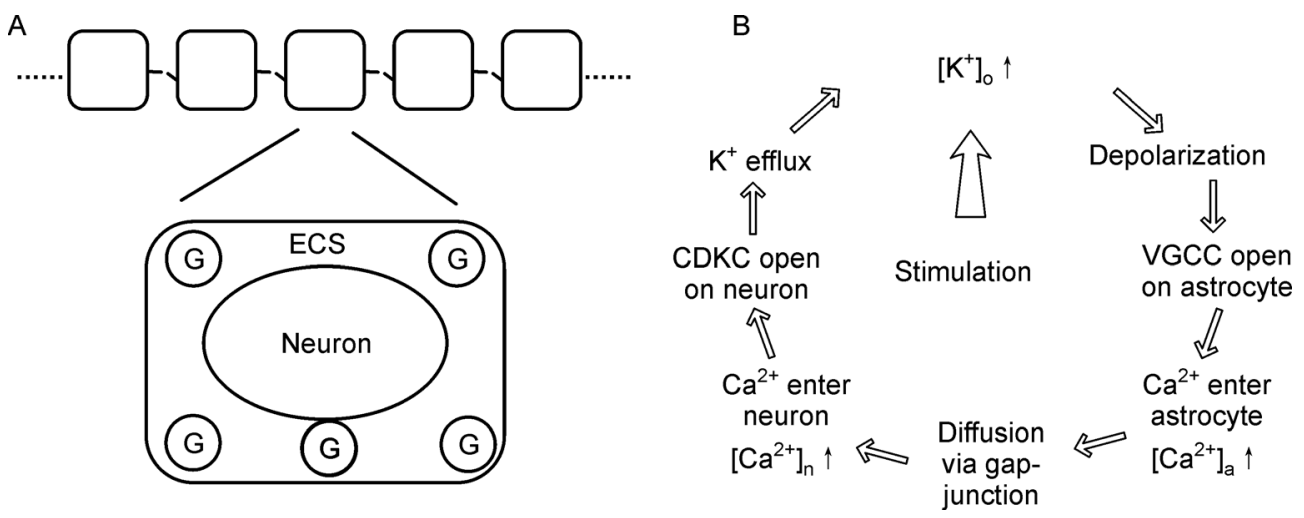

Figure 9. The conceptual model and processes of CSD. (A) Cortical tissue is simplified as a one dimensional continuum with three compartments: neurons, glial cells (i.e., 'G' in the figure) and the extracellular space (ECS). (B) shows the primary processes during CSD. VGCC represents as the voltage-gated calcium channels in astrocytes, and CDKC represents as the calciumdependent potassium channels in neurons. The stimulation is injection of $\mathrm{KCl}$ solution in this model. 
Table 2. Biological meanings and values of the parameters.

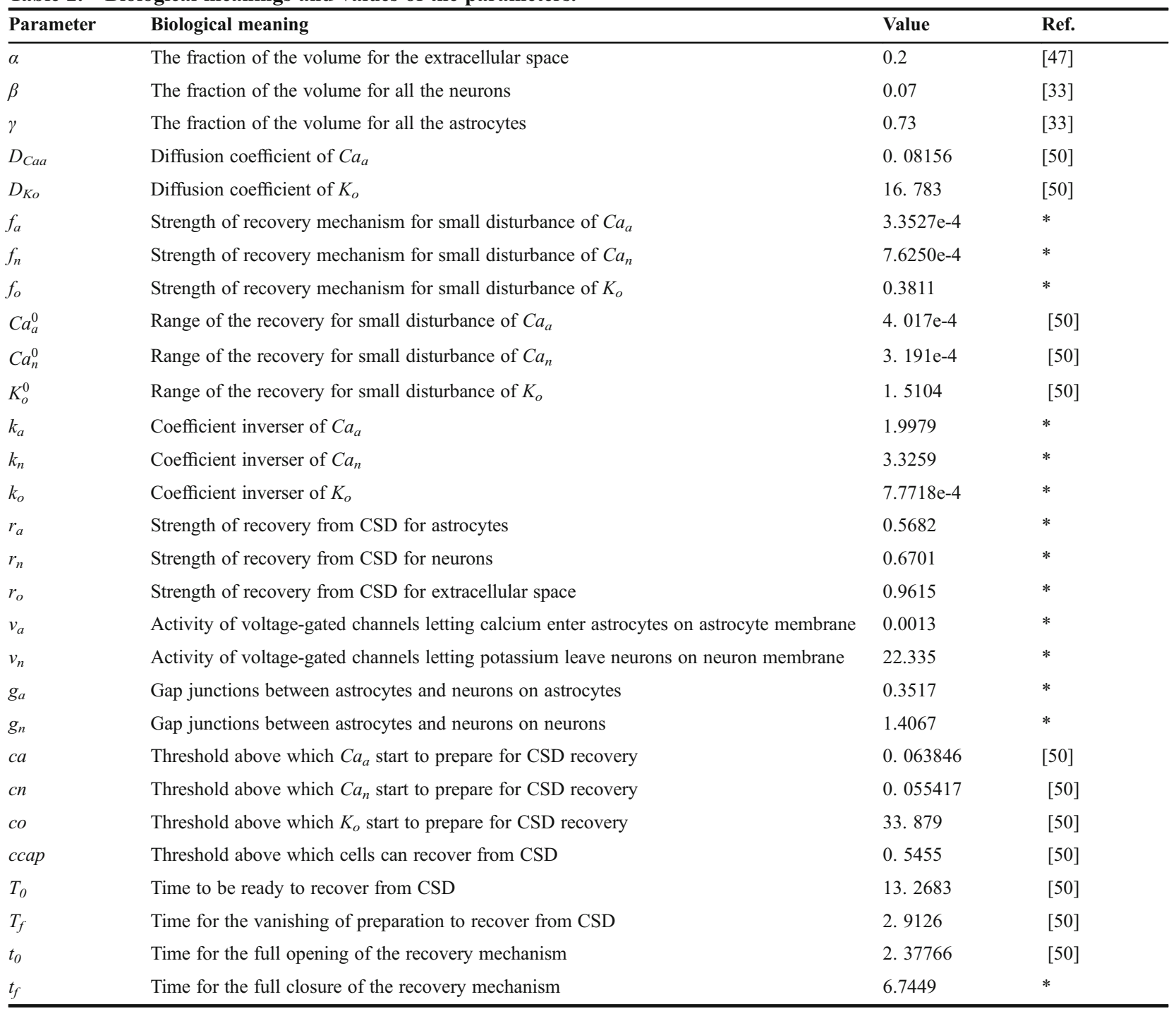

*Values come from our calculation obeying the rule proposed by Chapuisat $[35,50]$.

into discrete steps with temporal precision of $1 \mathrm{~s}$ and spatial precision of $0.2 \mathrm{~mm}$ respectively. Since high extracellular potassium was considered as the driving force sustaining CSD propagation [53,54], an injection of $\mathrm{KCl}$ with concentration $20 \mathrm{mM}$ around $1 \mathrm{~mm}$ area was used to initiate CSD. For transient stimulation, the stimulation was only kept for $1 \mathrm{~s}$. The continuous stimulation was kept for an assigned time (e.g., $3600 \mathrm{~s}$ ) in this paper. The propagation dynamics of CSD waves was represented as the change of extracellular potassium concentration.

\section{ACKNOWLEDGMENTS}

The authors are very grateful to Dr. G. Chapuisat for helps in constructing the computational model. This work was supported by Ministy of Science and Technology's high-tech (863) Projects (Nos. 2012AA020401 and 2012AA02A602).

\section{COMPLIANCE WITH ETHICS GUIDELINES}

The authors Bing Li, Shangbin Chen, Dong Yu and Pengcheng Li declare that they have no conflict of interests.

This article does not contain any studies with human or animal subjects performed by any of the authors.

\section{REFERENCES}

1. Leão, A. A. P. (1944) Spreading depression of activity in the cerebral cortex. J. Neurophysiol., 7, 359-390

2. Leão, A. A. P. (1944) Pial circulation and spreading depression of activity in the cerebral cortex. J. Neurophysiol., 7, 391-396

3. Leão, A. A. P. (1951) The slow voltage variation of cortical spreading depression of activity. Electroencephalogr. Clin. Neurophysiol., 3, 315321

4. Kraig, R. P. and Nicholson, C. (1978) Extracellular ionic variations during spreading depression. Neuroscience, 3, 1045-1059

5. Martins-Ferreira, H., Nedergaard, M. and Nicholson, C. (2000) 
Perspectives on spreading depression. Brain Res. Rev., 32, 215-234

6. Somjen, G. G. (2001) Mechanisms of spreading depression and hypoxic spreading depression-like depolarization. Physiol. Rev., 81, 1065-1096

7. Mayevsky, A. and Weiss, H. R. (1991) Cerebral blood flow and oxygen consumption in cortical spreading depression. J. Cereb. Blood Flow Metab., 11, 829-836

8. Li, P. C., Chen, S. B., Luo, W. H. and Luo, Q. M. (2003) Simultaneous imaging intrinsic optical signals and cerebral vessel responses during cortical spreading depression in rats. Proc. SPIE, 5254, 145-149

9. Ducros, A., Tournier-Lasserve, E. and Bousser, M. G. (2002) The genetics of migraine. Lancet Neurol., 1, 285-293

10. Bolay, H., Reuter, U., Dunn, A. K., Huang, Z., Boas, D. A. and Moskowitz, M. A. (2002) Intrinsic brain activity triggers trigeminal meningeal afferents in a migraine model. Nat. Med., 8, 136-142

11. Dahlem, M. A., Engelmann, R., Löwel, S. and Müller, S. C. (2000) Does the migraine aura reflect cortical organization? Eur. J. Neurosci., $12,767-770$

12. Kager, H., Wadman, W. J. and Somjen, G. G. (2000) Simulated seizures and spreading depression in a neuron model incorporating interstitial space and ion concentrations. J. Neurophysiol., 84, 495-512

13. Gorji, A. (2001) Spreading depression: a review of the clinical relevance. Brain Res. Rev., 38, 33-60

14. Revett, K., Ruppin, E., Goodall, S. and Reggia, J. A. (1998) Spreading depression in focal ischemia: a computational study. J. Cereb. Blood Flow Metab., 18, 998-1007

15. Mies, G., Lijima, T. and Hossmann, K. A. (1993) Correlation between peri-infarct DC shifts and ischaemic neuronal damage in rat. Neuroreport, 4, 709-711

16. Piper, R. D., Lambert, G. A. and Duckworth, J. W. (1991) Cortical blood flow changes during spreading depression in cats. Am. J. Physiol., 261, H96-H102

17. Fabricius, M., Jensen, L. H. and Lauritzen, M. (1993) Microdialysis of interstitial amino acids during spreading depression and anoxic depolarization in rat neocortex. Brain Res., 612, 61-69

18. James, M. F., Smith, M. I., Bockhorst, K. H. J., Hall, L. D., Houston, G. C., Papadakis, N. G., Smith, J. M., Williams, A. J., Xing, D., Parsons, A. A., et al. (1999) Cortical spreading depression in the gyrencephalic feline brain studied by magnetic resonance imaging. J. Physiol., 519, 415-425

19. Kuge, Y., Hasegawa, Y., Yokota, C., Minematsu, K., Hashimoto, N., Miyake, Y. and Yamaguchi, T. (2000) Effects of single and repetitive spreading depression on cerebral blood flow and glucose metabolism in cats: a PET study. J. Neurol. Sci., 176, 114-123

20. Ebersberger, A., Schaible, H. G., Averbeck, B. and Richter, F. (2001) Is there a correlation between spreading depression, neurogenic inflammation, and nociception that might cause migraine headache? Ann. Neurol., 49, 7-13

21. Miura, R. M., Huang, H. X. and Wylie, J. J. (2013) Mathematical approaches to modeling of cortical spreading depression. Chaos, 23, 046103

22. Miura, R. M., Huang, H. X. and Wylie, J. J. (2007) Cortical spreading depression: An enigma. Eur. Phys. J. Spec. Top., 147, 287-302.

23. Ayata, C. and Lauritzen, M. (2015) Spreading depression, spreading depolarizations, and the cerebral vasculature. Physiol. Rev., 95, 953993

24. Bockhorst, K. H., Smith, J. M., Smith, M. I., Bradley, D. P., Houston, G. C., Carpenter, T. A., Hall, L. D., Papadakis, N. G., Parsons, A. A.,
Huang, C. L., et al. (2000) A quantitative analysis of cortical spreading depression events in the feline brain characterized with diffusionweighted MRI. J. Magn. Reson. Imaging, 12, 722-733

25. Bradley, D. P., Smith, J. M., Smith, M. I., Bockhorst, K. H. J., Papadakis, N. G., Hall, L. D., Parsons, A. A., James, M. F. and Huang, C. L. H. (2002) Cortical spreading depression in the feline brain following sustained and transient stimuli studied using diffusionweighted imaging. J. Physiol., 544, 39-56

26. Smith, J. M., Bradley, D. P., James, M. F. and Huang, C. L. (2006) Physiological studies of cortical spreading depression. Biol. Rev. Camb. Philos. Soc., 81, 457-481

27. Chen, S., Li, P., Luo, W., Gong, H., Zeng, S. and Luo, Q. (2006) Timevarying spreading depression waves in rat cortex revealed by optical intrinsic signal imaging. Neurosci. Lett., 396, 132-136

28. Chen, S. B., Gong, H., Zeng, S. Q., Luo, Q. M. and Li, P. C. (2007) Elicitation interval dependent spatiotemporal evolution of cortical spreading depression waves revealed by optical intrinsic signal imaging. Proc. SPIE, 6445, 64450R-1-11.

29. Buchheim, K., Weissinger, F., Siegmund, H., Holtkamp, M., Schuchmann, S. and Meierkord, H. (2002) Intrinsic optical imaging reveals regionally different manifestation of spreading depression in hippocampal and entorhinal structures in vitro. Exp. Neurol., 175, 76-86

30. Eiselt, M., Giessler, F., Platzek, D., Haueisen, J., Zwiener, U. and Röther, J. (2004) Inhomogeneous propagation of cortical spreading depression-detection by electro- and magnetoencephalography in rats. Brain Res., 1028, 83-91

31. Brand, S., Fernandes de Lima, V. M. and Hanke, W. (1998) Pharmacological modulation of the refractory period of retinal spreading depression. Naunyn Schmiedebergs Arch. Pharmacol., 357, 419-425

32. Teixeira, H. Z., Almeida, A. C., Infantosi, A. F., Rodrigues, A. M., Costa, N. L. and Duarte, M. A. (2008) Identifying essential conditions for refractoriness of Leão's spreading depression-computational modeling. Comput. Biol. Chem., 32, 273-281

33. Li, B., Chen, S., Li, P., Luo, Q. and Gong, H. (2014) Refractory period modulates the spatiotemporal evolution of cortical spreading depression: a computational study. PLoS One, 9, e84609

34. Kager, H., Wadman, W. J. and Somjen, G. G. (2002) Conditions for the triggering of spreading depression studied with computer simulations. J. Neurophysiol., 88, 2700-2712

35. Godukhin, O. V. and Obrenovitch, T. P. (2001) Asymmetric propagation of spreading depression along the anteroposterior axis of the cerebral cortex in mice. J. Neurophysiol., 86, 2109-2111

36. Lin, J. H., Weigel, H., Cotrina, M. L., Liu, S., Bueno, E., Hansen, A. J., Hansen, T. W., Goldman, S. and Nedergaard, M. (1998) Gap-junctionmediated propagation and amplification of cell injury. Nat. Neurosci., 1, 494-500

37. Ba, A. M., Guiou, M., Pouratian, N., Muthialu, A., Rex, D. E., Cannestra, A. F., Chen, J. W. and Toga, A. W. (2002) Multiwavelength optical intrinsic signal imaging of cortical spreading depression. J. Neurophysiol., 88, 2726-2735

38. Tuckwell, H. C. (2008) Mathematical modeling of spreading cortical depression: spiral and reverberating waves. AIP Conf. Proc., 1028, 4664.

39. Bennett, M. R., Farnell, L. and Gibson, W. G. (2008) A quantitative model of cortical spreading depression due to purinergic and gapjunction transmission in astrocyte networks. Biophys. J., 95, 56485660 
40. Chapuisat, G., Dronne, M. A., Grenier, E., Hommel, M., Gilquin, H. and Boissel, J. P. (2008) A global phenomenological model of ischemic stroke with stress on spreading depressions. Prog. Biophys. Mol. Biol., 97, 4-27

41. Makarova, J., Ibarz, J. M., Canals, S. and Herreras, O. (2007) A steadystate model of spreading depression predicts the importance of an unknown conductance in specific dendritic domains. Biophys. J., 92, 4216-4232

42. Obrenovitch, T. P., Chen, S. and Farkas, E. (2009) Simultaneous, live imaging of cortical spreading depression and associated cerebral blood flow changes, by combining voltage-sensitive dye and laser speckle contrast methods. Neuroimage, 45, 68-74

43. Tuckwell, H. C. and Miura, R. M. (1978) A mathematical model for spreading cortical depression. Biophys. J., 23, 257-276

44. Shapiro, B. E. (2001) Osmotic forces and gap junctions in spreading depression: a computational model. J. Comput. Neurosci., 10, 99 120

45. Dronne, M. A., Boissel, J. P. and Grenier, E. (2006) A mathematical model of ion movements in grey matter during a stroke. J. Theor. Biol., 240, 599-615

46. Richter, F., Ebersberger, A. and Schaible, H. G. (2002) Blockade of voltage-gated calcium channels in rat inhibits repetitive cortical spreading depression. Neurosci. Lett., 334, 123-126
47. Lian, X. Y. and Stringer, J. L. (2004) Astrocytes contribute to regulation of extracellular calcium and potassium in the rat cerebral cortex during spreading depression. Brain Res., 1012, 177-184

48. Margineanu, D. G. and Klitgaard, H. (2006) The connexin 36 blockers quinine, quinidine and mefloquine inhibit cortical spreading depression in a rat neocortical slice model in vitro. Brain Res. Bull., 71, 23-28

49. Chapuisat, G. (2007) Discussion of a simple model of spreading depressions. ESAIM: Proc., 18, 87-98

50. Nicholson, C. and Syková, E. (1998) Extracellular space structure revealed by diffusion analysis. Trends Neurosci., 21, 207-215

51. Li, B., Chen, S., Zeng, S., Luo, Q. and Li, P. (2012) Modeling the contributions of $\mathrm{Ca}^{2+}$ flows to spontaneous $\mathrm{Ca}^{2+}$ oscillations and cortical spreading depression-triggered $\mathrm{Ca}^{2+}$ waves in astrocyte networks. PLoS One, 7, e48534

52. Ding, H., Chen, S., Zeng, S., Zeng, S., Liu, Q. and Luo, Q. (2008) Computation and visualization of spreading depression based on reaction-diffusion equation with recovery. Proc. SPIE, 7280.

53. Obrenovitch, T. P. and Zilkha, E. (1995) High extracellular potassium, and not extracellular glutamate, is required for the propagation of spreading depression. J. Neurophysiol., 73, 2107-2114

54. Enger, R., Tang, W., Vindedal, G. F., Jensen, V., Johannes Helm, P., Sprengel, R., Looger, L. L. and Nagelhus, E. A. (2015) Dynamics of ionic shifts in cortical spreading depression. Cereb. Cortex, 2015, 1-8 